\title{
Correspondence
}

\section{Regularization of RIF Blind Image Deconvolution}

Michael K. Ng, Robert J. Plemmons, and Sanzheng Qiao

\begin{abstract}
Blind image restoration is the process of estimating both the true image and the blur from the degraded image, using only partial information about degradation sources and the imaging system. Our main interest concerns optical image enhancement, where the degradation often involves a convolution process. We provide a method to incorporate truncated eigenvalue and total variation regularization into a nonlinear recursive inverse filter (RIF) blind deconvolution scheme first proposed by Kundur and Hatzinakos. Tests are reported on simulated and optical imaging problems.
\end{abstract}

Index Terms-Blind image deconvolution, circulant matrix, inverse filter, regularization.

\section{INTRODUCTION}

A fundamental issue in image restoration is blur removal in the presence of observation noise. In the important case where the blurring operation is spatially invariant, then the basic restoration computation involved is simply a deconvolution process that faces the usual difficulties associated with ill-conditioning in the presence of noise [2]. The image observed from a shift invariant linear blurring process, such as an optical system, is described by how the system blurs a point source of light into a larger image. The image of a point source is called the point spread function PSF, which we denote by $h$. The observed image $g$ is then the result of convolving the PSF $h$ with the "true" image, say $f$. This blurring process is represented by the convolution equation $g=h \star f$. The standard deconvolution problem is to recover the image $f$ given the observed image $g$ and the blurring operator $h$. There is much interest in removing blur and noise degradations from 1-D chemical spectra, as well as two-dimensional (2-D) images from microscopes, telescopes, and scintigrams [2]. The PSF of an imaging system can sometimes be described by a mathematical formula. More often, the PSF must be estimated empirically. Empirical estimates of the PSF can sometimes be obtained by imaging a relatively bright, isolated point source. In astro-imaging the point source might be a natural guide star or a guide star artificially generated using range-gated laser backscatter, e.g, [12]. Notice here that the PSF $h$ as well as the image may be degraded by noise.

In many applications data corresponding to $h$ is not completely known. Blind deconvolution is the process of estimating both the true image $f$ and the blur $h$ from the degraded image $g$. The purpose of this paper is to incorporate regularization into and refine a nonlinear

Manuscript received July 14, 1997; revised October 21, 1998. M. K. Ng's work was supported in part by the Research Grant Council under Grant HKU 7147/99P and HKU CRCG Grants 10201939 and 10202720. R. J. Plemmons' work was supported by the Air Force Office of Scientific Research and by the National Science Foundation. S. Qiao's work was supported by the Natural Sciences and Engineering Council of Canada. The associate editor coordinating the review of this manuscript and approving it for publication was Prof. Stephen E. Reichenbach.

M. K. Ng is with the Department of Mathematics, University of Hong Kong, Hong Kong (e-mail: mng@maths.hku.hk.).

R. J. Plemmons is with the Department of Computer Science, Wake Forest University, Winston-Salem, NC 27109 USA.

S. Qiao is with the Department of Computing and Software, McMaster University, Hamilton, Ont. L8S 4L7, Canada.

Publisher Item Identifier S 1057-7149(00)04868-5. recursive inverse filter blind deconvolution method first proposed by Kundur and Hatzinakos [8]-[10]. They call their scheme the nonnegativity and support constrained, recursive inverse filtering method (NAS-RIF). In this paper, we apply two regularization techniques to NAS-RIF. First, we apply an inexpensive eigenvalue truncation regularization to the filter. Second, we provide the user the option of applying total variation regularization to the estimated image. We call our approach the nonnegativity and support regularized recursive inverse filter (NAS-RRIF) algorithm. Note that the word "regularized" applies to both regularization techniques. The inverse filter and both regularization schemes are described in Section II. Numerical tests are reported in Section III on some simulated and optical imaging problems, and a comparison is made with the NAS-RIF algorithm.

\section{ITERATIVE BLIND DECONVOLUTION}

For simplicity, we develop our method the one-dimensional (1-D) restoration problem. Extensions to the 2-D image case are easily derived. Consider an image vector $f=\left(f_{1}, \cdots, f_{n}\right)^{T}, f_{i} \geq 0$, for $1 \leq i \leq n$, and a blurring filter with length $2 m+1$, represented by a $(2 m+1)$-by-1 vector $h=\left(h_{-m}, \cdots, h_{0}, \cdots, h_{m}\right)^{T}$. Assuming $2 m<n$, we can write the observed image $g$ as the convolution of $h$ and $f$. In matrix form, we have

$$
g=h \star f=\left(\begin{array}{cccc}
h_{-m} & & & 0 \\
\vdots & \ddots & & \\
h_{m} & \ddots & \ddots & \\
& \ddots & \ddots & h_{-m} \\
& & \ddots & \vdots \\
0 & & & h_{m}
\end{array}\right)\left(\begin{array}{c}
f_{1} \\
f_{2} \\
\vdots \\
f_{n-1} \\
f_{n}
\end{array}\right)=H f .
$$

Assuming the background color is represented by 0 , we can embed $H$ in a square circulant matrix and correspondingly embed $f$ in an $(n+2 m)$-by-1 vector by padding zeros. Then (1) is equivalent to

$$
g=C \tilde{f}=C\left(\begin{array}{c}
\tilde{f}_{a} \\
\tilde{f}_{b} \\
\tilde{f}_{c}
\end{array}\right)=C\left(\begin{array}{l}
0 \\
f \\
0
\end{array}\right)
$$

where $C$, the square and circulant matrix, is given by

$$
\left(\begin{array}{ccc|ccc|ccc}
h_{0} & \cdots & h_{-m+1} & h_{-m} & & 0 & h_{m} & \cdots & h_{1} \\
h_{1} & \cdots & h_{-m+2} & \vdots & \ddots & & 0 & \ddots & \vdots \\
\vdots & \ddots & \vdots & \vdots & & h_{-m} & \vdots & \ddots & h_{m} \\
h_{m} & \ddots & h_{1} & \vdots & & \vdots & h_{-m} & \ddots & \vdots \\
0 & \ddots & \vdots & \vdots & & \vdots & \vdots & \ddots & 0 \\
\vdots & \ddots & h_{m} & \vdots & & \vdots & h_{-1} & \ddots & h_{-m} \\
h_{-m} & \ddots & \vdots & h_{m} & & \vdots & \vdots & \ddots & \vdots \\
\vdots & \ddots & 0 & & \ddots & \vdots & h_{m-2} & \cdots & h_{-1} \\
h_{-1} & \cdots & h_{-m} & 0 & & h_{m} & h_{m-1} & \cdots & h_{0}
\end{array}\right) .
$$

Equation (2) is called the extended sequence convolution form of (1) and leads to the extended sequence deconvolution method [6]. Here, (2) is often preferable to (1), because its coefficient matrix $C$ is a square 
circulant matrix, which has many desirable properties. For example, if $H$ in (1) has full column rank, then $C$ is invertible and its inverse is also circulant and $C^{-1}$ can be efficiently computed using the fast Fourier transform (FFT), yielding the extended sequence deconvolution method for computing $f$.

In blind deconvolution, we estimate both $f$ and $H$ in (1) given the observed image $g$. To make the problem better determined, we impose some constraints. First, we assume the nonnegativity of pixel values in $f$. This is called the nonnegativity constraint, and can be incorporated into deconvolution computations in [2]. Second, since the size of $f$ differs from that of the given $g$, we assume the support, the size $n$ of $f=\tilde{f}_{b}$ in (2), is known. Thus, the structure of $C$ in (2) can be determined by $n$ and the size of $g$. Third, in the models (1) and (2), we assume there is no noise present. Finally, recall that we assume the background color is 0 as indicated in (2). Assuming that $C$ is invertible, we can formulate the blind deconvolution problem as follows.

- Find $C$ (or the first column of $C$ ) such that $\tilde{f}=C^{-1} g$ with $\tilde{f}_{a}=\tilde{f}_{c}=0$ and $\tilde{f}_{b} \geq 0$ in (2).

Some iterative blind deconvolution methods begin with a knowledge-based (nonnegativity, finite support) estimate for $f$, "deconvolve" $g=h \star f$ to estimate $h$, and then iterate in an alternating fashion to improve the estimates for both $f$ and $h$ [3], [5], [14]. These methods involve variations of direct, rather than inverse, filtering. Kundur and Hatzinakos [8]-[10], however, have recently presented a novel blind deconvolution method using recursive inverse filtering (RIF). Inverse filters are easier to implement and avoid certain inversion procedures, thus reducing the computational complexity.

The method by Kundur and Hatzinakos uses a variable finite impulse response (FIR) filter of length $2 p+1$, where $p$ is an estimate for $m$ in $H$ in (1), characterized by its parameter vector: $u=\left(u_{-p}, \cdots, u_{0}, \cdots, u_{p}\right)^{T}$, and the process iterates with respect to $u$. The observed image $g$ is the input to the filter. The output is the convolution of $u$ and $g: y=u \star g$, which is then passed through a nonlinear filter which maps $y$ to $\tilde{y}$ such that $\tilde{y}$ has a finite support, say $n$, and is nonnegative. In other words, the nonlinear filter sets any component which is either outside the finite support or negative, to zero. The resulting vector $\tilde{y}$ is an estimate for the true nonnegative image with support $n$, i.e., $\tilde{y} \approx \tilde{f}$. The parameter vector $u$ for our FIR filter is determined by minimizing the error $\|y-\tilde{y}\|_{2}^{2}$. In order to avoid a trivial null vector $u$, an additional term $\gamma\left(\sum_{i=-p}^{p} u_{i}-1\right)^{2}$ is incorporated into the objective function, where $\gamma \geq 0$. Specifically, the objective function is

$$
J(u)=\|y-\tilde{y}\|_{2}^{2}+\gamma\left(e^{T} u-1\right)^{2}
$$

where $e=(1, \cdots, 1)^{T}$. An optimal FIR filter is found by minimizing the above objective function $J(u)$. It is proved in [10] that $J(u)$ is convex. Thus a global minimum exists and a variety of numerical optimization algorithms can be used to compute $u$ minimizing $J(u)$. Kundur and Hatzinakos [9], [10] use a nonlinear conjugate gradient method to search for the minimum. Thus they call their algorithm the nonnegativity and support constraints recursive inverse filter (NAS-RIF).

\section{A. Regularizing Iterative Blind Deconvolution}

Continuous deconvolution can be modeled as an integral equation of the first kind (an ill-posed inverse problem [4]). It is well-known that deconvolution algorithms can be extremely sensitive to noise [2]. For example, if the noise is additive, then the blurring process can be represented by a convolution equation of the form $g=h \star f+\eta$, which, by (2), is equivalent to $g=C \tilde{f}+\eta$. Assuming that $C$ is invertible, we have $C^{-1} g=\tilde{f}+C^{-1} \eta$. In applications arising from integral equations of the first kind, the ill-conditioning of $C$ stems from the wide range of the magnitudes of its eigenvalues [4]. Therefore, excess amplification of the noise at small eigenvalues of $C$ can occur. Since any realistic signal processing problem involves noise, it is necessary to incorporate regularization into deconvolution to stabilize the computation [4]. Regularization methods attempt to alleviate sensitivity to the noise by "filtering" out eigen-components of the solution belonging to the noise subspace. For some iterative methods, it has been established that early termination of the iterations accomplishes this regularization effect. That is, the eigen-components of the signal subspace are reconstructed in the first (possibly many) iterations and, after reaching a certain approximate restoration, the components in the noise subspace begin to be reconstructed. It is at this point, where the noise begins to contaminate the reconstruction, that the iterations are halted (see, e.g., [4]). The simulations by Kundur and Hatzinakos [10] show that their NAS-RIF algorithm is sensitive to noise and, in fact, amplifies noise. The regularization method they employ is that of truncated iterations. The main problem with using early termination of the image restoration iterations as a regularization tool is the following. Due to varying activity levels, parts of the image may converge at different rates, leading to introduction of unwanted artifacts [2].

We describe an alternative regularization approach for the NAS-RIF algorithm. Our proposed method is referred to as the nonnegativity and support constraints regularized recursive inverse filter (NAS-RRIF). The idea is to first apply regularization to the inverse filter by using an inexpensive eigenvalue truncation scheme. Using the circulant matrix $C$ representation, we propose to use a $k$-by- $k$ circulant matrix, which we call $S$, to approximate the inverse of $C$, where $k$ is the size of the blurred image $g$. Specifically, we let $S$ be the $k$-by- $k$ circulant matrix with first column

$$
\left(s_{0}, \cdots, s_{p}, 0, \cdots, 0, s_{-p}, \cdots, s_{-1}\right)^{T}, \quad 1 \leq p<\frac{k}{2}
$$

where $p$ is an estimate for $m$ in $H$ in (1), and denote $S=$ $\operatorname{Circ}\left[\left(s_{0}, \cdots, s_{p}, 0, \cdots, 0, s_{-p}, \cdots, s_{-1}\right)^{T}\right]$. Thus, the FIR filter performs a multiplication of the circulant matrix $S$ and the observed image $g$. This multiplication can be implemented efficiently using the FFT. The output $y=S g$ is sent to the nonlinear filter and mapped to $\tilde{y}$ satisfying the nonnegativity and support constraints. Then the error $z=y-\tilde{y}$ is measured and fed back to compute an adjustment to the FIR parameter vector to reduce the value of the objective function. We choose eigenvalue truncation method for regularization because $S$ is circulant, its eigenvalues can be efficiently computed using the FFT. Then the vector $\hat{s}=F\left(s_{0}, \cdots, s_{p}, 0, \cdots, 0, s_{-p}, \cdots, s_{-1}\right)^{T}$ contains the eigenvalues of $S$. Here $F$ is the discrete Fourier matrix. To incorporate regularization into deconvolution to stabilize the computation, we need to penalize the small, in magnitude, eigenvalues of the convolution matrix $C$. Equivalently, in our inverse filter, we suppress the large, in magnitude, eigenvalues of the deconvolution matrix $S$ as follows. We first apply a nonlinear filter represented by a $k$-by- $k$ projection matrix $Q$

$$
\tilde{s}=Q \hat{s}
$$

where $Q=\operatorname{diag}\left(q_{i}\right)$ and

$$
q_{i}=\left\{\begin{array}{ll}
1, & \text { if }\left|\hat{s}_{i}\right| \leq \tau \\
0, & \text { otherwise; }
\end{array} \quad \text { for } 1 \leq i \leq k\right.
$$

where $\tau$ is a predetermined tolerance. In other words, the nonlinear filter characterized by $Q$ sets all the eigenvalues whose absolute values are greater than $\tau$ to zero. Effectively, the regularization is achieved by truncating the small eigenvalues of $S^{-1}$, which is an estimate for the convolution matrix $C$, as discussed in [7] and [11]. Then we measure the error $v=\hat{s}-\tilde{s}=(I-Q) \hat{s}$ and incorporate this error into the objective function $J(u)$ given by (3). Specifically, let $s=$ 


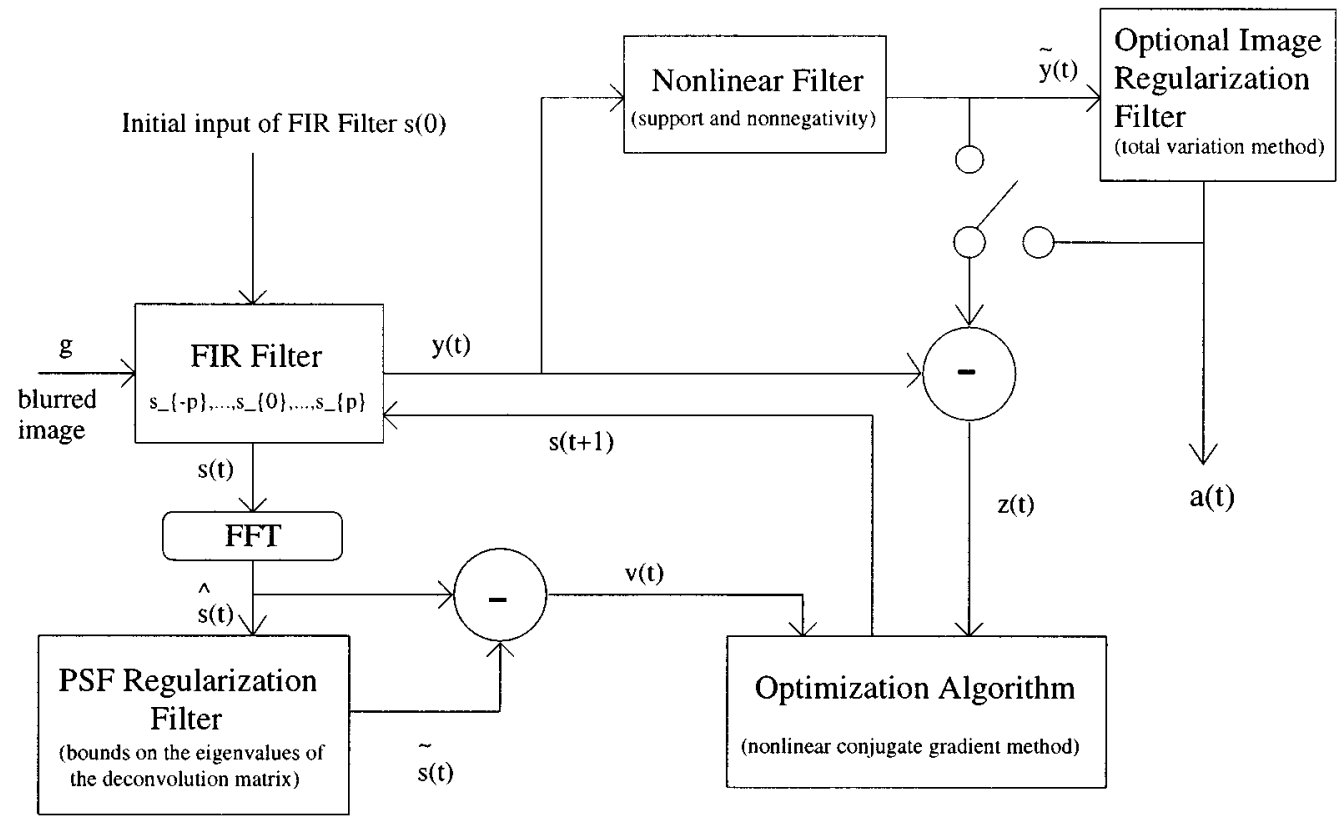

Fig. 1. NAS-RRIF Algorithm

$\left(s_{-p}, \cdots, s_{-1}, s_{0}, \cdots, s_{p}\right)^{T}$ be the filter parameter vector, then our regularized objective function is reformulated now in terms of $s$ as

$$
J_{r e g}(s) \equiv\|z\|_{2}^{2}+\mu\|v\|_{2}^{2}+\gamma\left(e^{T} s-1\right)^{2}
$$

where

$$
z=y-\tilde{y}, \quad v=\hat{s}-\tilde{s} .
$$

The parameter $\mu$ in (5) controls the degree of regularization. The filter parameter vector $s$ is determined by minimizing our new objective function (5). Fig. 1, which also involves regularization of the image calculation as described in Section II-B, gives an overview of our overall scheme.

Now we consider the gradient of $J_{r e q}$. For the first term in (5), the vector $\tilde{y}$ in $z=y-\tilde{y}$ is a projection of $y$. Denote $\tilde{y}=P y$, then $P$ is a diagonal matrix with 0 and 1 entries. Thus $z=(I-P) y$, where $y=S g$ is the output of the filter. Note that the circulant matrix $S$ is determined by the filter parameter vector $s$. For the second term in (5), the error vector $v=(I-Q) \hat{s}$ where $Q$ is also a diagonal matrix with 0 and 1 entries. Also, note that from the definitions of $\hat{s}$ and $s$

$$
\hat{s}=E s \quad \text { where } \quad E=F\left(\begin{array}{cc}
0 & I_{p+1} \\
0 & 0 \\
I_{p} & 0
\end{array}\right) .
$$

Using some algebraic manipulation, we can show that the gradient of $J_{r e g}(s)$ is

$$
\nabla J_{\text {reg }}(s)=2 G z+2 \mu \operatorname{Re}\left(E^{H} v\right)+2 \gamma\left(e^{T} s-1\right) e
$$

where $G$ is a $(2 p+1)$-by- $k$ row-circulant matrix with its first row: $\left(g_{p+1}, g_{p+2}, \cdots, g_{k}, g_{1}, \cdots, g_{p}\right)$. Since $G$ is row-circulant and $F$ is the $k$-by- $k$ Fourier matrix, the gradient can be computed efficiently. Furthermore, we can show that $J_{r e g}(s)$ is convex since the Hessian of the objective function, given by

$$
\nabla^{2} J_{r e g}(s)=2 G(I-P) G^{T}+2 \mu \operatorname{Re}\left(E^{H}(I-Q) E\right)+2 \gamma e e^{T}
$$

is positive semidefinite. Thus, a global minimum exists and a variety of numerical optimization algorithms can be used to compute an $s$ minimizing $J_{r e g}(s)$. In our numerical examples, a nonlinear conjugate gradient method is used to search for the global minimum. Usually, the initial vector $s$ is set to all zeros with a unit spike in the middle, see [9] and [10].

\section{B. Regularizing the Estimated Image}

Our method for regularizing the estimated image involves total variation minimization, which has been studied extensively in recent years, e.g., [3] and [13]. We choose this approach because it is especially effective in preserving sharp edges without penalizing smooth images. Also, in our application, this approach preserves nonnegativity of the image. The disadvantages, however, include the complexity level in solving total variation minimization problems, and the fact that minimizing the variation can sometimes cause a loss of fine detail in the image [4]. For these reasons, we leave the incorporation of total variation regularization of the estimated image as a user option in our NAS-RRIF scheme (see Fig. 1).

Let $\tilde{a}$ be the nonnegative segment of size $n$ at the center of $\tilde{y}$, i.e., $\tilde{a}=\left(\begin{array}{lll}0 & I_{n} & 0\end{array}\right) \tilde{y}$. We perform the regularization by solving for $a=$ $\left(a_{1}, \cdots, a_{n}\right)^{T}$ in the following penalized least squares minimization problem:

$$
\min _{a}\left\{\frac{1}{2}\|\tilde{a}-a\|_{2}^{2}+\alpha \sum_{i=1}^{n-1} \sqrt{\left|a_{i+1}-a_{i}\right|^{2}+\beta}\right\}
$$

where $\alpha>0$ controls the degree and $\beta(\geq 0)$ controls the variability of the penalty term [13]. When $\beta=0$, we have the usual total variation minimization. Suppose that $a$ is the solution of above minimization problem. The gradient of the associated objective function at $a$ is $a+\alpha A_{\beta}(a) a-\tilde{a}$, where $A_{\beta}(a)$ is a symmetric and tridiagonal matrix. Thus, the minimizer $a$ is the solution of the following equation $\left(I_{n}+\alpha A_{\beta}(a)\right) a=\tilde{a}$. We make two observations: first, $I_{n}+\alpha A_{\beta}(a)$ is an M-matrix (see e.g., [1]). Thus, the entries of the inverse of $\left(I_{n}+\right.$ $\left.\alpha A_{\beta}(a)\right)$ are nonnegative. Second, $a$ is the result of two consecutive mappings on the filter output $y$, first nonnegativity and support and then total variation regularization. These two observations ensure the 

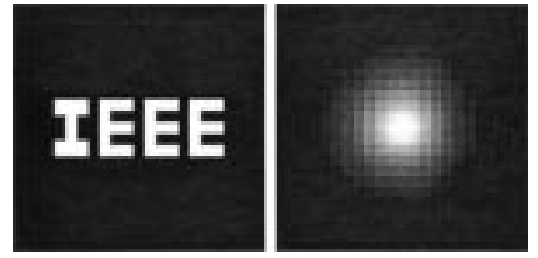

Fig. 2. (left) Original image and (right) point spread function.

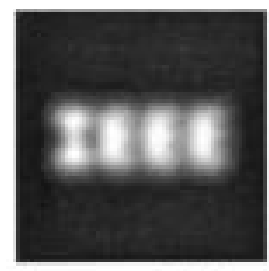

Rel. error $=0.6751$

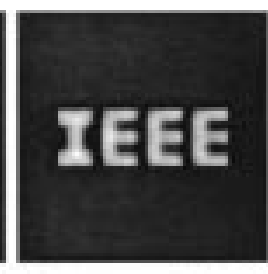

Rel. error $=0.3991$

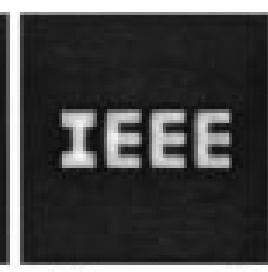

Rel. error $=0.2987$
Fig. 3. Degraded image with SNR $=70 \mathrm{DB}$ (left), NAS-RRIF restoration using $p=8$ (filter length $=17$ ), $\gamma=0.1, \mu=100$ and $\tau=100$ with (middle) 65 iterations, and (right) after post-processing the image estimate.

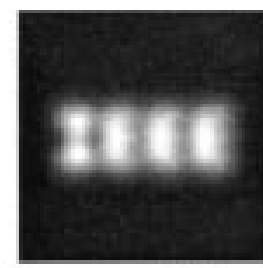

Rel. error $=0.6825$

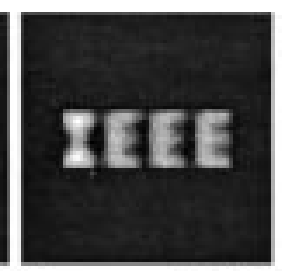

Fel. error $=0,5481$

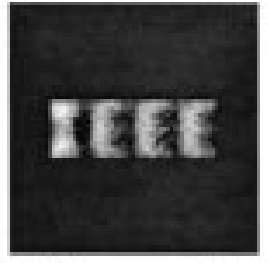

Rel. error $=0.6219$
Fig. 4. Degraded image with SNR $=20$ DB (left), NAS-RRIF restoration using $p=2$ (filter length $=5$ ), $\gamma=0.1$ and $\mu=0$ (no regularization) with 11 iterations (middle) and 18 iterations (right)

nonnegativity of $a$. Notice that the regularized image estimate $a$ can replace $\tilde{y}$ as an input to compute the error vector $z$ in the recursive inverse filter algorithm. The switch in Fig. 1 indicates this option. We remark that this image regularization method can also be incorporated into the NAS-RIF by Kundur and Hatzinakos [9], [10] to possibly improve their restored image.

Finally, we note that the 1-D results of Section II extend in a natural way to 2-D image blind deconvolution.

\section{NUMERICAL EXAMPLES IN OPTICAL IMAGING}

In this section, we present numerical tests of data samples to illustrate the effectiveness of our NAS-RRIF approach to image restoration by blind deconvolution.

Synthetic Data: The first data example consists of a $45 \times 25$ synthetically generated binary text image of the letters "IEEE" as shown in Fig. 2 (left). To obtain a blurred image, we used a Gaussian-type blurring filter with size $23 \times 23$, shown in Fig. 2 (right), and convolved it with the original image. The blurred image is also polluted by Gaussian noise so that the resulting observed image has $\mathrm{SNR}=70 \mathrm{DB}$ and $\mathrm{SNR}$ $=20 \mathrm{DB}$, as shown in Fig. 3 (left) and Fig. 4 (left), respectively.

For our NAS-RRIF algorithm, the best restoration is achieved at 73 iterations and 65 iterations when SNR $=70 \mathrm{DB}$ and SNR $=20 \mathrm{DB}$, respectively. We see from Fig. 3 (middle) and Fig. 5 (left) that a visually appealing result is obtained. For comparison purposes, the NAS-RRIF algorithm without filter regularization [i.e., $\mu=0$ in (5)], which is effectively NAS-RIF, is applied to the degraded image with a high noise level $(\mathrm{SNR}=20 \mathrm{DB})$. The algorithm without filter regularization can be viewed as the variant of the NAS-RIF method suggested by Kundur

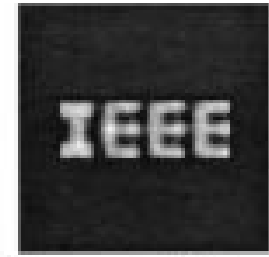

Rel, error $=0.4398$

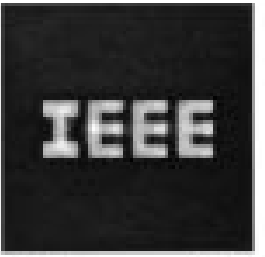

Ret. error $=0.3789$
Fig. 5. NAS-RRIF restoration using $p=8$ (filter length $=17$ ), $\gamma=0.1$, $\mu=100$, and $\tau=100$ (left) with 73 iterations and (right) after post-processing the image estimate with total variation regularization.
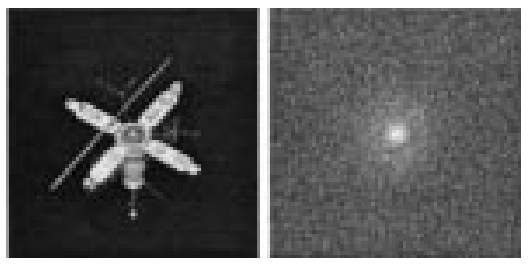

Fig. 6. (left) Original satellite image and (right) guide star image.
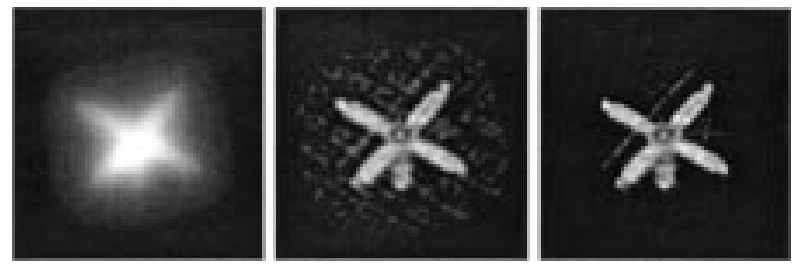

Fig. 7. (left) Observed image, (middle) restored image with the overestimated support, and (right) restored image with the exact support using the guide star image to initialize NAS-RRIF.

and Hatzinakos [9], [10]. We see that when SNR $=20 \mathrm{DB}$, the method converges to a solution [Fig. 4 (middle) with 11 iterations], but then their scheme exhibits noise amplification on subsequent iterations [Fig. 4 (right) with 18 iterations]. In addition, the corresponding relative errors

$$
\|f-y\|_{2} /\|f\|_{2}
$$

are shown at the bottom of the restored images, where $f$ and $y$ are the original and restored images Our tests thus indicate that the NAS-RRIF algorithm can effectively recover images even in the presence of high noise levels. We also remark that we choose the parameter $\tau$ by experimentation. Some formal methods for choosing regularization parameter can be found in [7] and [11].

We also illustrate that the post-processing of the image estimate given by NAS-RRIF algorithm can effectively regularize the restored image. We see from Figs. 3 (right) and 5 (right) that our blind deconvolution restored image post-processing option, described in Section II-B, and using total variation regularization, is useful in removing clutter, while preserving sharp edges.

Ground-Based Telescope Data: We consider a $256 \times 256$ image with irregular boundaries. This model problem data was obtained from the U.S. Air Force Phillips Laboratory, Lasers and Imaging Directorate, Kirtland Air Force Base, NM. The model has been used for testing various image restoration algorithms, e.g., [3] and [11]-[13].

Specifically, the true object is an ocean reconnaissance satellite, which is shown in Fig. 6 (left). A computer simulation algorithm at Phillips Laboratory was used to produce a degraded image of the satellite, shown in Fig. 7 (left), as would be observed from a modern ground-based telescope equipped with adaptive-optics controlled deformable mirrors [12]. The satellite was modeled as being $12 \mathrm{~m}$ 
in length and in an orbit $500 \mathrm{~km}$ above the surface of the earth. The simulated charge-coupled device (CCD) for forming the image was a 65536 pixel square array. CCD root-mean-square read-out noise variance was fixed at 15 microns/pixel to reflect a realistic state-of-the-art detector. In actual field experiments, several hundred measurement are averaged to reduce the effects of noise. In this example, the SNR of the blurred image is around $30 \mathrm{DB}$. The guide star observed under similar circumstances is shown in Fig. 6 (right). Notice the blur and noise in the image of the guide star, resulting in a degraded PSF. In this example, we set the initial estimate of the 2-D convolution matrix $C$ with eigenvalues

$$
\tilde{\lambda}_{i}= \begin{cases}\lambda_{i}, & \text { if }\left|\lambda_{i}\right| \geq 0.005 \\ 1, & \text { if }\left|\lambda_{i}\right|<0.005\end{cases}
$$

where $\lambda_{i}$ are the eigenvalues of the estimated PSF given by the guide star image. The filter parameter vector is initialized by applying the 2-D inverse FFT to the vector of eigenvalues $1 / \lambda_{i}$. Computed restorations by our NAS-RRIF algorithm (four iterations, $\mu=1.2, \tau=120$, and $\gamma=4.5$ ) using the knowledge of the guide star are shown in Fig. 7. We also see that use of the observed guide star image as initialization and use of a good estimate of the support are useful in the blind deconvolution of the satellite image.

In summary, we have introduced regularization methods to the recursive inverse filter for blind deconvolution in [8]-[10]. First, we use the eigenvalue truncation scheme in the filter to regularize the inverse problem. Second, we choose total variation minimization to improve the image estimated by the filter. We have shown that the new objective function is convex and the total variation regularization preserves the nonnegativity. Preliminary numerical results indicate the effectiveness of the method.

\section{REFERENCES}

[1] A. Berman and R. J. Plemmons, Nonnegative Matrices in the Mathematical Sciences, 2nd ed. Philadelphia, PA: SIAM, 1994.

[2] K. Castleman, Digital Image Processing. Englewood Cliffs, NJ: Prentice-Hall, 1996.

[3] T. Chan and C. Wong, "Total variation blind deconvolution," IEEE Trans. Image Processing, vol. 7, pp. 370-375, Mar. 1998.

[4] H. Engl, M. Hanke, and A. Neubauer, Regularization of Inverse Problems. Norwell, MA: Kluwer, 1996.

[5] D. Fish, A. Brinicombe, and R. Pike, "Blind deconvolution by means of the Richardson-Lucy algorithm," J. Opt. Soc. Amer. A, vol. 12, pp. $58-65,1995$.

[6] R. Gonzalez and R. Woods, Digital Image Processing. Reading, MA: Addison-Wesley, 1992.

[7] M. Hanke, J. Nagy, and R. Plemmons, "Preconditioned iterative regularization for Ill-posed problems," in Numerical Linear Algebra and Scientific Computing, L. Reichel, A. Ruttan, and R. Varga, Eds. Berlin, Germany: de Guyter, 1993, pp. 141-163.

[8] D. Kundur, "Blind deconvolution of still images using recursive inverse filtering," M.A.Sc. thesis, Univ. Toronto, Toronto, Ont., Canada, 1995, submitted for publication.

[9] D. Kundur and D. Hatzinakos, "Blind image deconvolution," IEEE Signal Processing Mag., pp. 43-64, May 1996.

[10] D. Kundur and D. Hatzinakos, "A novel blind deconvolution scheme for image restoration using recursive filtering," IEEE Trans. Signal Processing, vol. 46, pp. 375-390, Feb. 1998

[11] J. Nagy, R. Plemmons, and T. Torgersen, "Iterative image restoration using approximate inverse preconditioning," IEEE Trans. Image Processing, vol. 5, pp. 1151-1162, July 1996.

[12] M. Roggemann and B. Welsh, Imaging Through Turbulence. Boca Raton, FL: CRC, 1996

[13] C. Vogel and M. Oman, "Iterative methods for total variation denoising," SIAM J. Sci. Comput., vol. 17, pp. 227-238, 1996.
[14] Y. You and M. Kaveh, "A regularization approach to joint blur identification and image restoration," IEEE Trans. Image Processing, vol. 5, pp. 416-427, 1996.

[15] D. Kundur and D. Hatzinakos, "Blind image deconvolution revisited," IEEE Signal Processing Mag., pp. 61-63, Nov. 1996.

\section{Roof-Edge Preserving Image Smoothing Based on MRFs}

\author{
Stan Z. Li
}

\begin{abstract}
A novel Markov random field (MRF) model is proposed for roof-edge (as well as step-edge) preserving image smoothing. Image surfaces containing roof-edges are represented by piecewise continuous polynomial functions governed by a few parameters. Piecewise smoothness constraint is imposed on these parameters rather than on the surface heights as is in traditional models for step-edges. In this way, roof edges are preserved without the necessity to deal with instable higher order derivatives.
\end{abstract}

Index Terms-Image smoothing, Markov random fields (MRF), maximum a posteriori (MAP), roof edge, smoothness.

\section{INTRODUCTION}

Image smoothing is aimed at removing corrupting noise and restoring true image surfaces. It is performed based on the smoothness constraint about image surfaces which assumes that certain physical properties in a neighborhood present some coherence and generally do not change abruptly. The smoothness is imposed on the image surface function by using a Markov random field (MRF) [1]-[3] or regularization [4], [5] formulation.

Edges contain important information for image analysis and an important issue in image smoothing is edge preserving. Two major types of edges are steps and roofs. Step-edge preserving smoothing has been well researched and there exist a number of successful models, such as the line process model [1] in the Markov random field (MRF) framework and the weak string and membrane models [5] in the regularization framework; further studies can be found in [6]-[10]. These models assume that the underlying surface has zero first order derivatives and are suitable for preserving step-edges but not for roof-edges. Higher order derivatives have to be dealt with for roof-edges, but such algorithms suffer from instability [5].

In this paper, a novel MRF representation is proposed in which first order piecewise smoothness (to explained in the main text) is used for roof-edge (as well as step-edge) preserving smoothing. Image surfaces are assumed to be a piecewise function governed by a few parameters. Roof discontinuities in the image surface function correspond to step discontinuities in some governing parameter functions. So, it suffices to preserve roof edges if the first order piecewise smoothness is imposed on the parameter functions rather than directly on the image surface function. Roof edges in the surface function, i.e. step edges in the parameter functions, are preserved as if step edges in the surface are preserved in the line process model. This extends the ability of first

Manuscript received March 5, 1996; revised July 22, 1999. This work was supported by Nanyang Technological University under Grants NTU-AcRF RG 43/95 and RG 51/97. The associate editor coordinating the review of this manuscript and approving it for publication was Dr. Andrew F. Laine.

The author is with Microsoft Research China, Beijing 100080, China (e-mail: szli@microsoft.com; http://www.research.microsoft.com/users/szli).

Publisher Item Identifier S 1057-7149(00)04697-2. 